\title{
A Non-Convex Economic Dispatch Problem with Point-Valve Effect Using a Wind-Driven Optimisation Approach
}

\author{
Nur Fariza Ramli ${ }^{1}$. Nor Azwan Mohamed Kamari ${ }^{1,2}$ (1) Syahirah Abd Halim ${ }^{1} \cdot$ Mohd Asyraf Zulkifley $^{1}$. \\ Mohd Saiful Mohd Sahri ${ }^{3} \cdot$ Ismail Musirin $^{4}$
}

Received: 25 May 2021 / Revised: 13 July 2021 / Accepted: 27 July 2021 / Published online: 9 August 2021

(c) The Korean Institute of Electrical Engineers 2021

\begin{abstract}
This study presents the efficiency of the wind-driven optimisation (WDO) approach in solving non-convex economic dispatch problems with point-valve effect. The best economic dispatch for a power system is one wherein the system can generate energy at a low cost. The calculation of the generating cost is subject to a number of constraints, such as the power demand for the entire system and the generation limit for each generator unit in the system. In addition, the system should also produce low power loss. The WDO optimisation technique is developed based on the concept of natural wind movement, which serves as a stabiliser to equalise the inequality of air pressure in the atmosphere. One major advantage of WDO over other techniques is its search accuracy. The proposed algorithm has been implemented in two systems, namely, the 10-generator and 40-generator systems. Both systems were tested in a Matlab environment. To highlight the capabilities of WDO, the results using this proposed technique are compared with the results obtained using flower pollination algorithm, moth flame optimisation, particle swarm optimisation and evolutionary programming techniques to determine the efficiency of the proposed approach in solving economic dispatch. The simulation results show the capability of WDO in determining the optimal power generation value with minimum generation cost and low rate of power loss.
\end{abstract}

Keywords Non-convex problem formulation · Wind driven optimisation · Flower pollination algorithm · Moth flame optimisation $\cdot$ Particle swarm optimisation $\cdot$ Evolutionary programming

\section{Introduction}

With the simultaneous increase of economic and population growth, the demand for electricity in a country is also expected to rise every year. However, in early 2020, the world was shocked by the outbreak of COVID-19, which was later declared a pandemic by the World Health Organisation (WHO). The spread of this pandemic has significantly

Nor Azwan Mohamed Kamari

azwank@ukm.edu.my

1 Department of Electrical, Electronic and Systems Engineering, Faculty of Engineering and Built Environment, Universiti Kebangsaan Malaysia, Bangi, Selangor, Malaysia

2 Institute of IR4.0, Universiti Kebangsaan Malaysia, Bangi, Selangor, Malaysia

3 Japan University Programme, Malaysia France Institute, Universiti Kuala Lumpur, Bangi, Selangor, Malaysia

4 Faculty of Electrical Engineering, Universiti Teknologi Mara, Shah Alam, Selangor, Malaysia affected power sectors worldwide. Most countries have declared lockdowns for prolonged periods, leading to a significant decline in energy consumption in the industrial and commercial sectors. However, as a result of this lockdown, the workforce has to manage their work from their own homes, paving the way for the so-called 'work-from-home' concept. This has resulted in increased energy demand in residential areas. Such developments have urged utility companies to improve their power grid networks and restructure their power generation scheduling.

Economic Dispatch (ED) schedules the power unit operations to meet a specific power demand whilst also imposing a minimum fuel cost. Categorised as a type of optimisation problem, ED solutions using optimisation can be divided into mathematical and heuristic techniques. Mathematical techniques include linear [1,2], quadratic [3] and mixedinteger [4] programming. These traditional ED solutions, however, are time-consuming, unable to solve non-linear cost functions and can only provide suboptimal solutions. Such disadvantages have led scientists to introduce heuristic 
approaches, in which ED problems can be categorised as smooth and non-smooth. In non-smooth problems, the impact of the valve system is considered in the power generation cost function. Both smooth and non-smooth problems have been successfully solved using heuristic techniques, as respectively reported in $[5,6]$ and $[7-10]$.

Artificial intelligence (AI) is widely used in the field of power systems. Amongst the techniques used are flower pollination algorithm (FPA) [11-14], moth flame optimisation (MFO) [15-18], particle swarm optimisation (PSO) [19-22] and evolutionary programming (EP) [23-26]. The FPA optimisation technique is developed based on the transfer of pollen from one flower to another on the same tree or another tree using natural pollinators, such as honey bees, birds, water or wind. MFO is inspired by moth navigation methods in nature and is known as a transverse orientation, ensuring that a moth is at a constant angle to the source of light, such as the moon and the candle flame for orientation. The PSO technique, which is also based on the movement of animal, is inspired by the feeding process of certain animals, such as swarming birds and schooling fish. EP is one of the evolutionary computing, which uses the models of biological evolutionary process such as mutation, for the solution of complex engineering problems. In the present study, a new metaheuristic-based method called wind driven optimisation (WDO) is introduced [27-30]. WDO is based on the concept of natural wind movement, which serves as a stabiliser to equalise the inequality of air pressure in the atmosphere. One advantage of WDO over other techniques is its search accuracy. In particular, its optimisation capabilities have been proven in various optimisation problems, such as economics delivery, engineering design and medical applications.

The current study proposes efficient techniques for calculating optimal, non-smooth power generation capacity based on power demand as well as the constraints of each generator unit, using the WDO optimisation approach. Test systems using 10- and 40-unit power generators are simulated using MATLAB. The objective function of this optimisation is to minimise the total cost of power generation. To determine the performance of the proposed technique, the results of using the WDO technique are compared with those obtained via FPA, MFO, PSO and EP.

The rest of the paper is organised into sections. Section 2 presents the non-convex problem formulation for ED. Section 3 explains the WDO algorithm. Section 4 provides the simulation results and discussions. Finally, Sect. 5 presents the conclusions.

\section{Formulation of Economic Dispatch}

In economic dispatch, energy generation planning is focused on coordinating energy production for each generation unit within a system. The amount of energy generated should exceed the power demand that has been set at an early stage. However, the production of high surplus energy will result in financial losses to energy operators. In addition, each generator unit has its own generating cost characteristics and different generating capacities. The generating unit cost calculation method can be divided into two formulations: convex and non-convex problem formulations.

In the fundamental concept of convex problem formulation, the cost function of each generator can be presented by a simple quadratic function presented by Eq. (1):

$\operatorname{Cos}_{c}\left(P_{i}\right)=a_{c, i}+b_{c, i} P_{i}+c_{c, i} P_{i}^{2}$,

where $\operatorname{Cost}_{c}\left(P_{i}\right)$ is the convex production cost of $P_{i}$ in $\$$ per hour, $P_{i}$ is the real power output of the $i$ th generator in MW, and $a_{c, i}, b_{c, i}$, and $c_{c, I}$ are three of the convex generation cost coefficients of $P_{i}$. The operating limits of $P_{i}$ can be presented as follows:

$P_{i, \min } \leq P_{i} \leq P_{i, \max }$

where the minimum and maximum operating limits of $P_{i}$ are $P_{i, \min }$ and $P_{i, \max }$, respectively.

According to its basic concept, the fuel cost function per generating unit is considered to increase quadratically; however, it cannot solve the ED problem practically without considering the point-valve effect. Such an effect can cause the generator input-output curve to become more complicated. This method of calculation based on the point-valve effect is called non-convex problem formulation. To model the pointvalve effect, a recurring rectified sinusoid contribution was added to the Eq. (1), represented by Eq. (3):

$$
\operatorname{Cos}_{n}\left(P_{i}\right)=a_{n, i}+b_{n, i} P_{i}+c_{n, i} P_{i}^{2}+\left|e_{n, i} \sin \left(f_{n, i}\left(P_{i, \min }-P_{i}\right)\right)\right|
$$

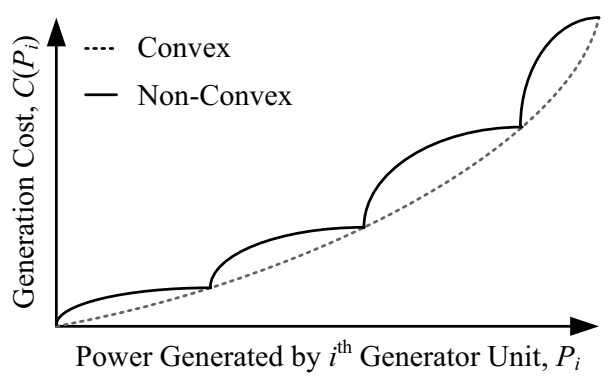

Fig. 1 Cost function according to the point-valve effect system (nonconvex) and without point-valve effect system (convex) 
where $\operatorname{Cost}_{n}\left(P_{i}\right)$ is the non-convex production cost of $P_{i}$, in $\$$ per hour. Five of the non-convex generation cost coefficients of $P_{i}$ are represented by $a_{n, i}, b_{n, i}, c_{n, i}, e_{n, i}$ and $f_{n, i}$, respectively. The obvious difference between these two problem formulations is the presence of a sinusoidal function on the cost equation for the non-convex problem formulation.

Figure 1 illustrates the convex problem formulations (systems without point-valve effects) and non-convex problem formulations (systems with point-valve effects). As can be seen, the cost rate for the system without the point-valve effect increases quadratically. In comparison, the cost rate for a system with a point-valve effect goes up and down (like a hill) along a quadratic line. This is the effect of the valve opening according to the requested power generation. In this study, the calculation of the generation cost of each generator unit will use the concept of non-convex problem formulation.

Based on the calculated production cost of every generator using non-convex problem formulations, the total production cost $\operatorname{Cost}_{T}$ with all $n$ generators in the system can be expressed as follows:

$\operatorname{Cos}_{T}=\operatorname{Cos}_{n}\left(P_{1}\right)+\operatorname{Cos}_{n}\left(P_{2}\right)+\cdots \cdots+\operatorname{Cos}_{n}\left(P_{n}\right)$.

The correlations among the total amount of power generated by all unit $P_{T}$, total power demand $P_{D}$, and power losses $P_{L}$ can be expressed as

$P_{T}=P_{1}+P_{2}+\cdots P_{n}=P_{D}+P_{L}$.

Apart from producing low generation costs, a good generation system also produces low $P_{L}$.

\section{Wind Driven Optimisation Techniques}

This study proposes a new technique based on wind movement called WDO. The basic optimisation method for WDO and the stopping criteria for finding the best generation cost are described in the next subtopic.

\subsection{Wind Driven Optimisation}

The concept of WDO was first developed by Zikri Bayraktar in 2010. It is based on the concept of natural wind movement, which serves as a stabiliser to equalise the inequality of air pressure in the atmosphere. Wind blows from a high- to a low-pressure area, according to a velocity that is directly proportional to the pressure gradient (the higher the pressure difference, the stronger the wind blows). Wind can be thought of as moving horizontally by assuming that the horizontal motion of the air is stronger than its vertical motion. The main principle behind the WDO technique is Newton's second law of motion:

$\rho \vec{a}=\sum \overrightarrow{F_{i}}$,

where $a$ is the acceleration vector, $\rho$ is the air density for an element with a very small volume and $F_{i}$ is the force acting on the mass. Air pressure, density and temperature are related by an equation according to the ideal gas law expressed as

$P=\rho R T$,

where $P, R$ and $T$ represent pressure, universal gas constant and temperature, respectively.

In Eq. (6), the main forces that cause the wind blowing in a direction to deviate from that direction can be broken down into four: pressure gradient force $\left(F_{P G}\right)$, frictional force $\left(F_{F}\right)$, gravitational force $\left(F_{G}\right)$ and coriolis force $\left(F_{C}\right)$. Their corresponding force equations are given below:

$\vec{F}_{P G}=-\nabla P \delta V$,

$\vec{F}_{C}=-2 \Omega \times \vec{u}$,

$\vec{F}_{G}=\rho \delta V \vec{g}$

$\vec{F}_{F}=-\rho \alpha \vec{u}$,

where $\nabla P$ is the pressure gradient, $\delta V$ is a very small volume of air, $\Omega$ represents the rotation of the earth, $u$ is the wind velocity vector and $g$ is the gravitational acceleration. The combination of Eqs. (6) and (8)-(11) produces an equation represented by Eq. (12) below:

$\rho \vec{u} \Delta t=-\nabla P \delta V-2 \Omega \times \vec{u}+\rho \delta V \vec{g}-\rho \alpha \vec{u}$.

A packet of air moving with the wind can be considered very small. The unit step of time, $\Delta t$ can also be considered equal to 1. The combination of Eqs. (7) and (12) produces the following equation:

$$
\begin{gathered}
\vec{u}_{\text {new }}=-g \vec{x}_{\text {old }}+(1-\alpha) \vec{u}_{\text {old }}+\left|1-\frac{P_{\max }}{P_{\text {old }}}\right| \\
R T\left(x_{\max }-x_{\text {old }}\right)-\frac{c u_{\text {old }}^{\text {other } \operatorname{dim}}}{P_{\text {old }}},
\end{gathered}
$$

where $u_{\text {new }}$ and $u_{\text {old }}$ are the updated velocity and the current velocity, respectively; $x_{\text {old }}$ and $x_{\max }$ are the current location and the highest-pressure location of the air pack, respectively; $P_{\max }$ and $P_{\text {old }}$ are the maximum pressure and the pressure at the current location, respectively; $T$ is the temperature; and $R, \alpha$ and $c$ are constants. 
The pressure value in Eq. (13) is usually high. Therefore, the new velocity estimations will also be high. This causes the performance level of the WDO to decrease. To solve this problem, the use of pressure values will be replaced by method based on ranking of air parcel [30]. The air parcel population is ranked in descending order based on its pressure value. Equation (13) is rearranged into the following equation:

$\vec{u}_{\text {new }}=-g \vec{x}_{\text {old }}+(1-\alpha) \vec{u}_{\text {old }}+\left|1-\frac{1}{k}\right| R T\left(x_{\max }-x_{\text {old }}\right)-\frac{c u_{\text {old }}^{\text {other } \operatorname{dim}}}{k}$.

Here, $k$ denotes the ranking between all air parcels $(k=1,2, \ldots, 20)$. The following equation is used to update the position of the air pack:

$\vec{x}_{\text {new }}=\vec{x}_{\text {old }}+\vec{u}_{\text {new }} \times \Delta t$.

The potential trajectories of the pressure gradient force, coriolis force, frictional force and gravitational force in WDO are shown in Fig. 2.

The flow chart of the optimisation process using the WOA technique is shown in Fig. 3. In this study, the ability of WOA to find the optimal generation cost value is compared with that of four other selected optimisation techniques, namely, FPA, MFO, PSO and EP. Detailed explanations of the FPA, MFO, PSO and EP approaches can be found in $[11,15,19]$ and [23], respectively.

\subsection{Stopping Criteria}

In this study, a total of 100 tests were simulated to obtain optimal results for each technique in every case. The

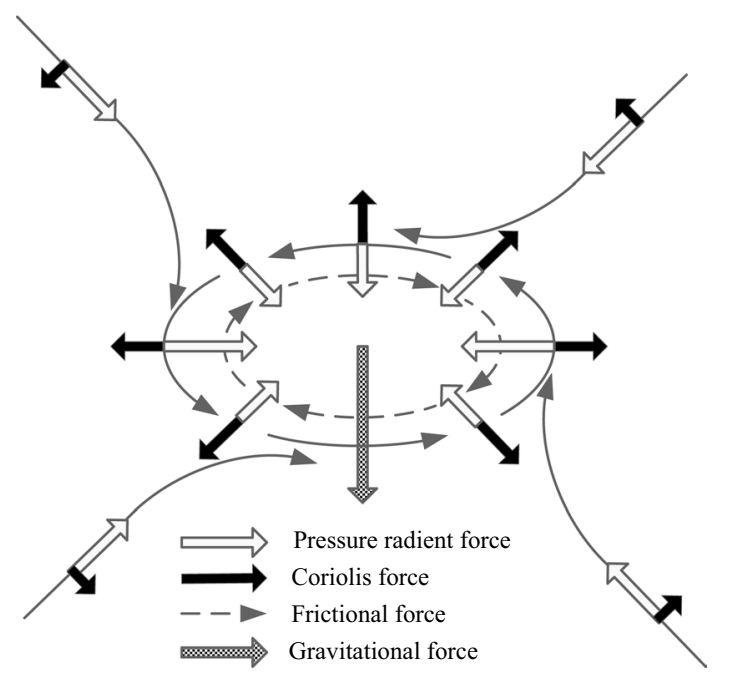

Fig. 2 Potential trajectories of the pressure gradient force, coriolis force, frictional force and gravitational force in WDO

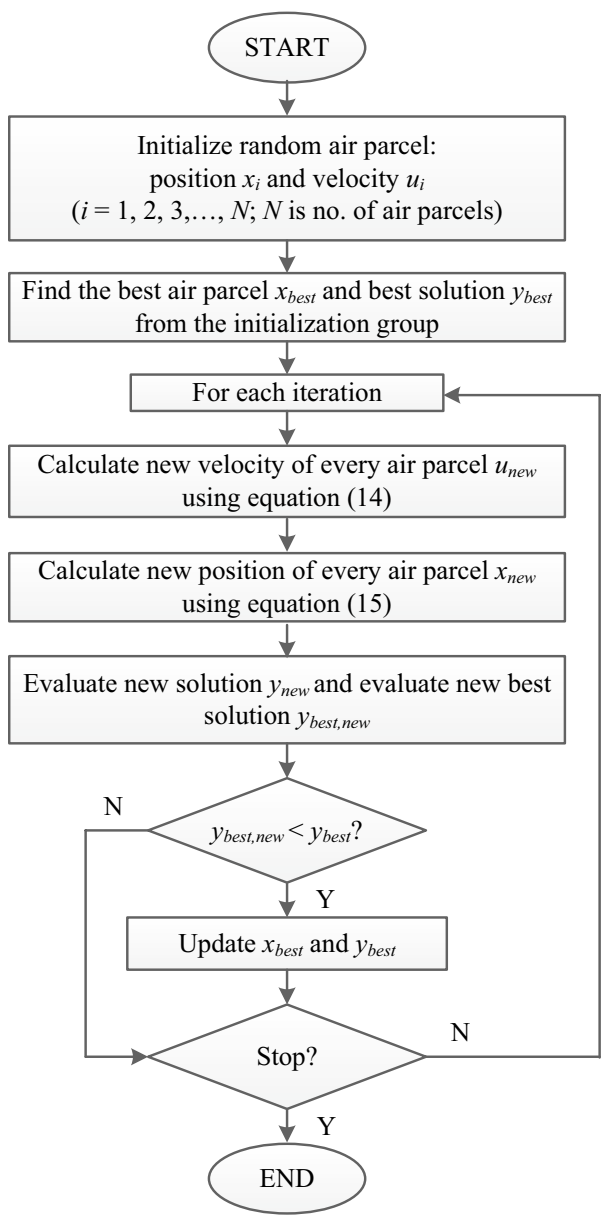

Fig. 3 The flow chart of the optimisation process using WOA

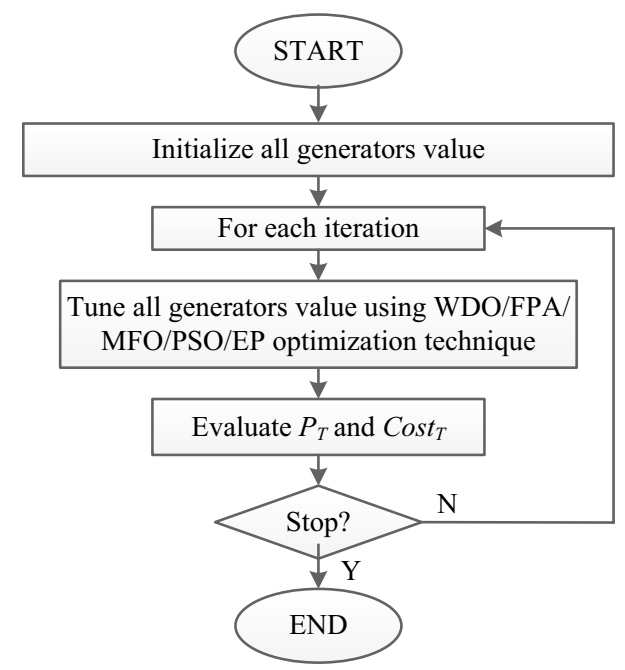

Fig. 4 The flow chart of the calculation process of $P_{T}$ and Cost $_{T}$ 
Table 1 Lists of test systems, cases and $P_{D}$ values

\begin{tabular}{lll}
\hline Test System & Cases & $P_{D}(\mathrm{MW})$ \\
\hline Ten Generators & Case 1 & 1400 \\
& Case 2 & 2050 \\
Forty Generators & Case 3 & 8700 \\
& Case 4 & 9650 \\
\hline
\end{tabular}

Table 2 List of parameter values used in WDO, FPA, MFO, PSO and EP

\begin{tabular}{llllll}
\hline WDO & \multicolumn{5}{c}{ MFO } \\
\hline$R T$ & RT coefficient & 35 & $\beta$ & Constant & 1 \\
$g$ & Gravity constant & 0.01 & $\tau$ & Random value & $(0,1]$ \\
$\alpha$ & Alpha & 0.9 & EP & & \\
$c$ & Coriolis effect & 0.95 & $\beta$ & Scaling factor & 0.05 \\
$v_{\max }$ & Maximum speed & 0.1 & PSO & & 0.05 \\
FPA & & & $\omega$ & Inertia weight & 0.5 \\
$p$ & Boundary value & 0.7 & $c_{1}$ & Accelerating coef- & 0.5 \\
& & & \multicolumn{5}{c}{ ficient } \\
$\varepsilon$ & Uniform distribution & $(0,1]$ & $c_{2}$ & $\begin{array}{c}\text { Accelerating coef- } \\
\text { ficient }\end{array}$ & 0.5 \\
& & & \multicolumn{5}{c}{} \\
\hline
\end{tabular}

maximum number of iterations per case is set at 300 iterations. Based on 100 tests for each of these cases, a consistent result can be obtained. Figure 4 shows the flow chart of the calculation process of $P_{T}$ and Cost $_{T}$.

As shown in Fig. 4, there are two criteria for the optimisation process to stop: (i) the difference between the maximum and minimum generation cost is less than $0.1 \%$ of minimum $\operatorname{Cost}_{T}$, and (ii) the current iteration is equal to the maximum number of iterations.

\section{Results and Discussions}

There were two test systems involved, namely, the 10- and 40-generator systems. Cases 1-A and 1-B were run using a 10-generator test system, whilst Cases 2-A and 2-B were run using a 40 -generator test system. The simulations were conducted using Matlab software in a computer with Intel (R) Core (TM) i5-8250U processing specifications. Table 1 illustrates all the test systems, cases and specific power demand $P_{D}$ values.

Table 2 shows the list of parameter values used in WDO, FPA, MFO, PSO and EP in this study.

Table 3 and Table 4 present the fuel cost coefficients $\left(a_{n, i}\right.$, $b_{n, i}, c_{n, i}, e_{n, i}$ and $\left.f_{n, i}\right)$ with minimum and maximum power limits $\left(P_{\min }\right.$ and $\left.P_{\max }\right)$ for each generator unit in ten-generator and forty-generator test systems, respectively [31].

Based on the simulations conducted, the iteration period taken by the EP technique is the lowest amongst the five approaches used, i.e. 10-20 iterations. For the WDO, FPA and PSO methods, the range of the number of iterations to complete the simulation is $50-100$ iterations. The MFO approach requires the highest number of iterations, i.e. 50-150 iterations.

Table 5 shows the optimisation results of 10 generator values; total power generation, $P_{T}$; and power loss, $P_{L}$ for Case 1 using the WDO, FPA, PSO, MFO and EP techniques. This value was obtained from 100 simulations conducted for each technique. If we compare the selected generation values for all 10 generators, there are very significant differences among these five approaches. Although the generation value for each of these generators is different, the majority of the methods achieve the same $P_{T}$ value as the prescribed $P_{D}$, which is $1400 \mathrm{MW}$. The $P_{L}$ results obtained using PSO are the best, resulting in zero power loss. This is followed by FPA, MFO and EP. WDO produces the highest power loss: $77.6 \mathrm{~kW}$. However, this power loss value is very minimal
Table 3 Power limits and fuel cost coefficients with valvepoint effect in ten-generator test system

\begin{tabular}{|c|c|c|c|c|c|c|c|}
\hline \multirow[t]{2}{*}{ Unit } & \multicolumn{2}{|l|}{ Power limits } & \multicolumn{5}{|c|}{ Fuel cost coefficients with valve-point effect } \\
\hline & $P_{\text {min }}(\mathrm{MW})$ & $P_{\max }(\mathrm{MW})$ & $\overline{a_{n, i}(\$ / h)}$ & $b_{n, i}(\$ / \mathrm{MWh})$ & $c_{n, i}\left(\$ /(\mathrm{MW})^{2} \mathrm{~h}\right)$ & $d_{n, i}(\$ / \mathrm{h})$ & $e_{n, i}(\mathrm{rad} / \mathrm{MW})$ \\
\hline 1 & 10 & 55 & 1000.403 & 40.5407 & 0.12951 & 33 & 0.0174 \\
\hline 2 & 20 & 80 & 950.606 & 39.5804 & 0.10908 & 25 & 0.0178 \\
\hline 3 & 47 & 120 & 900.705 & 36.5104 & 0.12511 & 32 & 0.0162 \\
\hline 4 & 20 & 130 & 800.705 & 39.5104 & 0.12111 & 30 & 0.0168 \\
\hline 5 & 50 & 160 & 756.799 & 38.5390 & 0.15247 & 30 & 0.0148 \\
\hline 6 & 70 & 240 & 451.325 & 46.1592 & 0.10587 & 20 & 0.0163 \\
\hline 7 & 60 & 300 & 1243.531 & 38.3055 & 0.03546 & 20 & 0.0152 \\
\hline 8 & 70 & 340 & 1049.998 & 40.3965 & 0.02803 & 30 & 0.0128 \\
\hline 9 & 135 & 470 & 1658.569 & 36.3278 & 0.02111 & 60 & 0.0136 \\
\hline 10 & 150 & 470 & 1356.659 & 38.2704 & 0.01799 & 40 & 0.0141 \\
\hline
\end{tabular}


Table 4 Power limits and fuel cost coefficients with valvepoint effect in forty-generator test system

\begin{tabular}{|c|c|c|c|c|c|c|c|}
\hline \multirow[t]{2}{*}{ Unit } & \multicolumn{2}{|l|}{ Power limits } & \multicolumn{5}{|c|}{ Fuel cost coefficients with valve-point effect } \\
\hline & $P_{\min }(\mathrm{MW})$ & $P_{\max }(\mathrm{MW})$ & $a_{n, i}(\$ / \mathrm{h})$ & $b_{n, i}(\$ / \mathrm{MWh})$ & $c_{n, i}\left(\$ /(\mathrm{MW})^{2} \mathrm{~h}\right)$ & $d_{n, i}(\$ / \mathrm{h})$ & $e_{n, i}(\mathrm{rad} / \mathrm{MW})$ \\
\hline 1 & 36 & 114 & 94.705 & 6.73 & 0.00690 & 100 & 0.084 \\
\hline 2 & 36 & 114 & 94.705 & 6.73 & 0.00690 & 100 & 0.084 \\
\hline 3 & 60 & 120 & 309.540 & 7.07 & 0.02028 & 100 & 0.084 \\
\hline 4 & 80 & 190 & 369.030 & 8.18 & 0.00942 & 150 & 0.063 \\
\hline 5 & 47 & 97 & 148.890 & 5.35 & 0.01140 & 120 & 0.077 \\
\hline 6 & 68 & 140 & 222.330 & 8.05 & 0.01142 & 100 & 0.084 \\
\hline 7 & 110 & 300 & 287.710 & 8.03 & 0.00357 & 200 & 0.042 \\
\hline 8 & 135 & 300 & 391.980 & 6.99 & 0.00492 & 200 & 0.042 \\
\hline 9 & 135 & 130 & 455.760 & 6.60 & 0.00573 & 200 & 0.042 \\
\hline 10 & 130 & 300 & 722.820 & 12.9 & 0.00605 & 200 & 0.042 \\
\hline 11 & 94 & 375 & 635.200 & 12.9 & 0.00515 & 200 & 0.042 \\
\hline 12 & 94 & 375 & 635.200 & 12.8 & 0.00569 & 200 & 0.042 \\
\hline 13 & 125 & 500 & 913.400 & 12.5 & 0.00421 & 300 & 0.035 \\
\hline 14 & 125 & 500 & 1760.400 & 8.84 & 0.00752 & 300 & 0.035 \\
\hline 15 & 125 & 500 & 1760.400 & 8.84 & 0.00752 & 300 & 0.035 \\
\hline 16 & 125 & 500 & 1760.400 & 8.84 & 0.00752 & 300 & 0.035 \\
\hline 17 & 220 & 500 & 647.850 & 7.97 & 0.00313 & 300 & 0.035 \\
\hline 18 & 220 & 500 & 649.690 & 7.95 & 0.00313 & 300 & 0.035 \\
\hline 19 & 242 & 550 & 647.830 & 7.97 & 0.00313 & 300 & 0.035 \\
\hline 20 & 242 & 550 & 647.810 & 7.97 & 0.00313 & 300 & 0.035 \\
\hline 21 & 254 & 550 & 785.960 & 6.63 & 0.00298 & 300 & 0.035 \\
\hline 22 & 254 & 550 & 785.960 & 6.63 & 0.00298 & 300 & 0.035 \\
\hline 23 & 254 & 550 & 794.530 & 6.66 & 0.00284 & 300 & 0.035 \\
\hline 24 & 254 & 550 & 794.530 & 6.66 & 0.00284 & 300 & 0.035 \\
\hline 25 & 254 & 550 & 801.320 & 7.10 & 0.00277 & 300 & 0.035 \\
\hline 26 & 254 & 550 & 801.320 & 7.10 & 0.00277 & 300 & 0.035 \\
\hline 27 & 10 & 150 & 1055.100 & 3.33 & 0.52124 & 120 & 0.077 \\
\hline 28 & 10 & 150 & 1055.100 & 3.33 & 0.52124 & 120 & 0.077 \\
\hline 29 & 10 & 150 & 1055.100 & 3.33 & 0.52124 & 120 & 0.077 \\
\hline 30 & 47 & 97 & 148.890 & 5.35 & 0.01140 & 120 & 0.077 \\
\hline 31 & 60 & 190 & 222.920 & 6.43 & 0.00160 & 150 & 0.063 \\
\hline 32 & 60 & 190 & 222.920 & 6.43 & 0.00160 & 150 & 0.063 \\
\hline 33 & 60 & 190 & 222.920 & 6.43 & 0.00160 & 150 & 0.063 \\
\hline 34 & 90 & 200 & 107.870 & 8.95 & 0.00010 & 200 & 0.042 \\
\hline 35 & 90 & 200 & 116.580 & 8.62 & 0.00010 & 200 & 0.042 \\
\hline 36 & 90 & 200 & 116.580 & 8.62 & 0.00010 & 200 & 0.042 \\
\hline 37 & 25 & 110 & 307.450 & 5.88 & 0.01610 & 80 & 0.098 \\
\hline 38 & 25 & 110 & 307.450 & 5.88 & 0.01610 & 80 & 0.098 \\
\hline 39 & 25 & 110 & 307.450 & 5.88 & 0.01610 & 80 & 0.098 \\
\hline 40 & 242 & 550 & 647.830 & 7.97 & 0.00313 & 300 & 0.035 \\
\hline
\end{tabular}

compared to $P_{D}$ (only representing $0.006 \%$ of the $P_{D}$ value) and is acceptable.

Table 6 shows the three optimal $\operatorname{Cost}_{T}$ generation cost values (best, worst and average) for Case 1. There are three optimal $\operatorname{Cost}_{T}$ values observed, namely, best, worst and average $\operatorname{Cost}_{T}$. From the perspective of generation cost based on the best $\operatorname{Cost}_{T}$ value, the WDO method yields the cheapest cost of $\$ 73,550.10$, followed by FPA, PSO, EP and finally
MFO. The cost difference between WDO and MFO is $\$ 1,616.40$, of which the use of WDO saves $2.15 \%$ compared to MFO. For the worst $\operatorname{Cost}_{T}$ value, PSO provides the cheapest generation cost, followed by FPA, WDO, EP and MFO. The PSO performance is also excellent from the perspective of the average $\operatorname{Cost}_{T}$, which is $\$ 74,046.00$, compared to MFO which yields the most expensive average $\operatorname{Cost}_{T}$ of $\$ 75,988.90$. 
Table 5 Optimal values of ten generators, $P_{T}$ and $P_{L}$ for Case 1

\begin{tabular}{lccccc}
\hline Generators & \multicolumn{5}{l}{ Power Output (MW) } \\
\cline { 2 - 6 } & WDO & FPA & PSO & EP & \multicolumn{1}{c}{ MFO } \\
\hline$P_{1}$ & 37.2460 & 32.8694 & 28.7449 & 13.9650 & 45.6974 \\
$P_{2}$ & 52.0730 & 42.1987 & 41.9311 & 41.2693 & 52.1088 \\
$P_{3}$ & 55.0002 & 61.4683 & 71.6364 & 94.6273 & 76.6114 \\
$P_{4}$ & 44.1233 & 35.3087 & 56.9393 & 89.4375 & 69.8739 \\
$P_{5}$ & 59.0050 & 57.5162 & 50.0248 & 50.0169 & 103.4620 \\
$P_{6}$ & 70.1490 & 71.9811 & 70.8618 & 89.1754 & 115.7997 \\
$P_{7}$ & 176.7250 & 184.3209 & 169.5514 & 90.9592 & 181.7518 \\
$P_{8}$ & 208.3102 & 194.2257 & 207.1486 & 155.3605 & 176.4693 \\
$P_{9}$ & 357.6415 & 368.1389 & 337.8918 & 422.3723 & 260.8406 \\
$P_{10}$ & 339.8043 & 351.9893 & 365.2700 & 352.8614 & 317.4054 \\
$P_{T}$ & 1400.1 & 1400.0 & 1400.0 & 1400.0 & 1400.0 \\
$P_{L}$ & 0.0776 & 0.0172 & 0.0000 & 0.0447 & 0.0203 \\
\hline
\end{tabular}

Table $6 \operatorname{Cost}_{T}$ (best, worst and average) for Case 1

\begin{tabular}{llllll}
\hline Cost $_{T}\left(\times 10^{3} \$\right)$ & WDO & FPA & PSO & EP & MFO \\
\hline Best Value & 73.5501 & 73.5681 & 73.5805 & 74.6649 & 75.1665 \\
Worst Value & 75.2377 & 75.1034 & 74.5993 & 76.2122 & 76.9685 \\
Average Value & 74.2104 & 74.1112 & 74.0460 & 75.7129 & 75.9889 \\
\hline
\end{tabular}

Table 7 Optimal values of ten generators for Case 2

\begin{tabular}{lrrrrr}
\hline Generators & \multicolumn{1}{l}{ Power Output (MW) } \\
\cline { 2 - 6 } & WDO & \multicolumn{1}{c}{ FPA } & \multicolumn{1}{l}{ PSO } & \multicolumn{1}{l}{ EP } & \multicolumn{1}{c}{ MFO } \\
\hline$P_{1}$ & 54.7399 & 53.1208 & 46.8454 & 31.2615 & 53.8569 \\
$P_{2}$ & 79.8961 & 77.2853 & 69.6440 & 78.4024 & 57.0711 \\
$P_{3}$ & 105.4016 & 118.7199 & 103.9364 & 74.0878 & 99.9383 \\
$P_{4}$ & 91.0725 & 94.7526 & 97.8784 & 128.9845 & 97.2851 \\
$P_{5}$ & 68.1580 & 81.2763 & 78.9019 & 76.5929 & 141.6828 \\
$P_{6}$ & 71.6061 & 75.1139 & 92.2134 & 150.7565 & 192.0888 \\
$P_{7}$ & 299.7558 & 296.6702 & 298.1497 & 284.8565 & 251.2110 \\
$P_{8}$ & 339.8989 & 338.3731 & 340.0000 & 315.9169 & 317.0608 \\
$P_{9}$ & 469.9720 & 467.8319 & 468.6216 & 452.7929 & 436.7844 \\
$P_{10}$ & 469.5987 & 447.2566 & 453.8091 & 456.4505 & 403.0211 \\
$P_{T}$ & 2050.1 & 2050.4 & 2050.0 & 2050.1 & 2050.0 \\
$P_{L}$ & 0.0996 & 0.4006 & 0.0000 & 0.1024 & 0.0002 \\
\hline \multicolumn{5}{c}{}
\end{tabular}

WDO ranks third in the five methods compared. In this Case 1, PSO gives the best and worst $\operatorname{Cost}_{T}$ difference. This indicates that in Case 1, PSO is able to maintain optimal value consistency in the 100 simulations run. This is followed by FPA, EP, WDO and MFO. Although less prominent for the worst and average Cost $_{T}$ values, WDO excelled in terms of best $\operatorname{Cost}_{T}$, thus making it the best optimisation approach amongst the five methods compared.
Table $8 \operatorname{Cost}_{T}$ (best, worst and average) for Case 2

\begin{tabular}{llllll}
\hline $\begin{array}{l}\operatorname{Cost}_{T}\left(\times 10^{3}\right. \\
\text { \$) }\end{array}$ & WDO & FPA & PSO & EP & MFO \\
\hline Best Value & 109.1962 & 109.4541 & 109.4660 & 110.5981 & 112.4619 \\
Worst Value & 111.2749 & 110.3995 & 110.4337 & 113.4144 & 114.7050 \\
$\begin{array}{l}\text { Average } \\
\text { Value }\end{array}$ & 109.9090 & 110.0390 & 110.1242 & 112.1201 & 113.8818 \\
\hline
\end{tabular}

The optimum values of 10 generators, $P_{T}$ and $P_{L}$ using WDO, FPA, PSO, EP and MFO for Case 2 are shown in Table 7. The five methods successfully tuned the values for each generator to produce the total generating power as set by $P_{D}$ which is $2,050 \mathrm{MW}$. PSO produced the lowest $P_{L}$ i.e. zero loss, followed by MFO, WDO, EP and FPA. The value of $P_{L}$ by FPA is $400.6 \mathrm{~kW}$, which is $0.02 \%$ of $P_{D}$. Despite the occurrence of power loss, the values given by the four techniques other than PSO are all extremely minimal and acceptable.

Table 8 tabulates the optimal Cost $_{T}$ (best, worst and average) based on 100 simulation tests for Case 2. On the one hand, for Case 2, the best and the average Cost $_{T}$ values by WDO, which are $\$ 109,162.90$ and $\$ 109,909.00$, respectively, are both considered excellent by producing the lowest values amongst the five methods. MFO, on the other hand, is an optimisation method that tunes the generator unit with the most expensive $\operatorname{Cost}_{T}$ amongst the best, worst and average optimal values. Amongst the five worst optimal values, WDO also provides the lowest generation cost values, followed by FPA, EP, PSO and MFO. Based on the results obtained for Case 2, WDO is the best approach among those compared in terms of tuning the generator value to produce the lowest generation cost.

Table 9 shows the optimum values of 40 generators using WDO for Case 3. As a proposed technique, the generator values calculated by WDO are tabulated in this table.

The values for total generation, $P_{T}$; power loss, $P_{L}$; and optimal fuel cost, $\operatorname{Cost}_{T}$ (best, worst and average) based on 100 simulation tests for the five optimisation techniques for Case 3 are shown in Table 10. As can be seen, WDO and FPA techniques generate total power values that are approximately similar to the power demand, $P_{D}$, which is $8,700 \mathrm{MW}$, with $P_{L}$ values at 39.9 and $28.1 \mathrm{~kW}$, respectively. This is followed by PSO, EP and MFO with $P_{L}$ values at 203.3, 871.4 and $954.3 \mathrm{~kW}$, respectively. The highest $P_{L}$ value is produced by the MFO, which is about $0.01 \%$ of the $P_{D}$ value-a minimal and acceptable value of power loss. From the best Cost $_{T}$ generation cost perspective, WDO produces the cheapest cost at $\$ 104,503.90$, followed by FPA, PSO, EP and MFO.

Table 10 also shows that the difference between the best $\operatorname{cost}_{T}$ and worst $\operatorname{cost}_{T}$ values (i.e. \$838), which is calculated 
Table 9 Optimal generator values using WDO for Case 3

\begin{tabular}{lccccccc}
\hline Gen & Values $(\mathrm{MW})$ & Gen & Values $(\mathrm{MW})$ & Gen & Values $(\mathrm{MW})$ & Gen & Values (MW) \\
\hline$P_{1}$ & 91.7379 & $P_{11}$ & 94.2034 & $P_{21}$ & 527.9771 & $P_{31}$ & 187.5987 \\
$P_{2}$ & 37.6354 & $P_{12}$ & 94.2941 & $P_{22}$ & 541.8251 & $P_{32}$ & 177.6737 \\
$P_{3}$ & 67.6266 & $P_{13}$ & 125.2984 & $P_{23}$ & 530.5006 & $P_{33}$ & 66.8977 \\
$P_{4}$ & 80.0122 & $P_{14}$ & 125.1635 & $P_{24}$ & 545.0939 & $P_{34}$ & 91.4794 \\
$P_{5}$ & 94.4204 & $P_{15}$ & 385.1753 & $P_{25}$ & 533.2426 & $P_{35}$ & 160.6316 \\
$P_{6}$ & 68.0171 & $P_{16}$ & 148.4389 & $P_{26}$ & 461.4438 & $P_{36}$ & 90.0110 \\
$P_{7}$ & 144.6910 & $P_{17}$ & 399.0958 & $P_{27}$ & 10.0316 & $P_{37}$ & 103.5682 \\
$P_{8}$ & 266.7026 & $P_{18}$ & 487.6544 & $P_{28}$ & 10.6505 & $P_{38}$ & 25.1094 \\
$P_{9}$ & 134.8687 & $P_{19}$ & 515.6547 & $P_{29}$ & 10.0184 & $P_{39}$ & 26.5686 \\
$P_{10}$ & 130.3725 & $P_{20}$ & 47.0359 & $P_{30}$ & 54.2192 & $P_{40}$ & 507.3999 \\
\hline
\end{tabular}

Table $10 P_{T}, P_{L}$ and Cost $_{T}$ (best, worst and average) for Case 3

Table 11 Optimal generator values using WDO for Case 4

\begin{tabular}{lccrrr}
\hline Generators & \multicolumn{4}{l}{ Power Output $(\mathrm{MW})$} & \multicolumn{1}{c}{} \\
\cline { 2 - 6 } & \multicolumn{1}{l}{ WDO } & FPA & \multicolumn{1}{l}{ PSO } & \multicolumn{1}{c}{ EP } & \multicolumn{1}{c}{ MFO } \\
\hline$P_{T}(\mathrm{MW})$ & 8700.0 & 8700.0 & 8700.2 & 8700.9 & 8701.0 \\
$P_{L}(\mathrm{MW})$ & 0.0399 & 0.0281 & 0.2033 & 0.8714 & 0.9543 \\
Best $\operatorname{cost}_{T}\left(\times 10^{3} \$\right)$ & 104.5039 & 104.7626 & 105.8389 & 112.7587 & 112.1324 \\
Worst $\operatorname{cost}_{T}\left(\times 10^{3} \$\right)$ & 112.0477 & 115.8665 & 109.6689 & 113.5967 & 113.9424 \\
Average $\operatorname{cost}_{T}\left(\times 10^{3} \$\right)$ & 108.3233 & 109.1284 & 107.0617 & 113.1129 & 115.5889 \\
\hline
\end{tabular}

\begin{tabular}{lccccccc}
\hline Gen & Values $(\mathrm{MW})$ & Gen & Values $(\mathrm{MW})$ & Gen & Values $(\mathrm{MW})$ & Gen & Values (MW) \\
\hline$P_{1}$ & 105.5497 & $P_{11}$ & 96.1000 & $P_{21}$ & 535.0146 & $P_{31}$ & 189.9561 \\
$P_{2}$ & 113.724 & $P_{12}$ & 132.9019 & $P_{22}$ & 546.4675 & $P_{32}$ & 170.6419 \\
$P_{3}$ & 64.3305 & $P_{13}$ & 125.0737 & $P_{23}$ & 524.4567 & $P_{33}$ & 75.3798 \\
$P_{4}$ & 136.119 & $P_{14}$ & 395.3443 & $P_{24}$ & 524.9659 & $P_{34}$ & 199.7569 \\
$P_{5}$ & 61.0432 & $P_{15}$ & 370.5575 & $P_{25}$ & 540.6237 & $P_{35}$ & 120.7023 \\
$P_{6}$ & 71.3441 & $P_{16}$ & 131.8998 & $P_{26}$ & 547.6057 & $P_{36}$ & 196.2475 \\
$P_{7}$ & 187.358 & $P_{17}$ & 493.3949 & $P_{27}$ & 11.4685 & $P_{37}$ & 94.2439 \\
$P_{8}$ & 284.0464 & $P_{18}$ & 477.9911 & $P_{28}$ & 13.7626 & $P_{38}$ & 109.7994 \\
$P_{9}$ & 134.5449 & $P_{19}$ & 549.8370 & $P_{29}$ & 18.7203 & $P_{39}$ & 108.9533 \\
$P_{10}$ & 241.832 & $P_{20}$ & 538.0521 & $P_{30}$ & 92.0219 & $P_{40}$ & 518.4186 \\
\hline
\end{tabular}

Table $12 P_{T}, P_{L}$, and $\operatorname{Cost}_{T}$ (best, worst and average) for Case 4

\begin{tabular}{lccrrr}
\hline Generators & \multicolumn{4}{l}{ Power Output $(\mathrm{MW})$} & \multicolumn{1}{l}{} \\
\cline { 2 - 6 } & WDO & FPA & \multicolumn{1}{l}{ PSO } & \multicolumn{1}{l}{ EP } & \multicolumn{1}{c}{ MFO } \\
\hline$P_{T}$ & 9850.3 & 9850.1 & 9850.1 & 9850.5 & 9850.1 \\
$P_{L}$ & 0.2515 & 0.1171 & 0.1207 & 0.4850 & 0.1134 \\
Best $\operatorname{cost}_{T}\left(\times 10^{3} \$\right)$ & 117.8404 & 118.9734 & 119.6477 & 125.9508 & 126.8486 \\
Worst $\operatorname{cost}_{T}\left(\times 10^{3} \$\right)$ & 125.0225 & 125.5121 & 121.4457 & 129.4023 & 130.3201 \\
Average $\operatorname{cost}_{T}\left(\times 10^{3} \$\right)$ & 122.4021 & 122.4920 & 122.4960 & 128.5443 & 128.6714 \\
\hline
\end{tabular}

using EP, is the smallest amongst the five methods. This indicates that for Case 3, the values based on the EP technique are the most consistent values for the entire 100 simulations conducted. Meanwhile, FPA has the largest best and worst $\operatorname{cost}_{T}$ difference of $\$ 1,110.39$. Despite providing the most consistent value, EP provides the most expensive $\operatorname{cost}_{T}$ of the five techniques for this case. Therefore, the best technique that provides the cheapest $\operatorname{cost}_{T}$ is WDO, followed by FPA, PSO and MFO. 


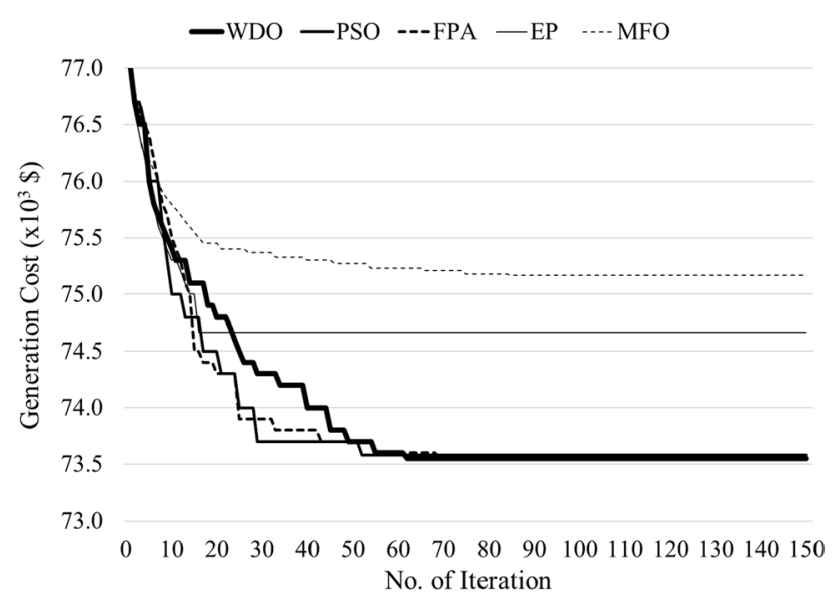

Fig. 5 The flow chart of the calculation process of $P_{T}$ and Cost $_{T}$

The optimum values of 40 generators (Set 2) using WDO for Case 4 are tabulated in Table 11.

The optimal $P_{T}, P_{L}$ and $\operatorname{Cost}_{T}$ values (best, worst and average) for Case 4 are shown in Table 12. As can be seen, all techniques are capable of achieving the $P_{D}$ set at $9850 \mathrm{MW}$. Of the five methods used, MFO has the lowest $P_{L}$ of $113.4 \mathrm{~kW}$, followed by FPA, PSO and WDO. EP tuned the highest $P_{L}$ at $485.0 \mathrm{~kW}$, which is $0.005 \%$ of the $P_{D}$ value. This indicates that all the $P_{L}$ values produced by the five optimisation methods in this case are minimal and acceptable. Despite giving the lowest $P_{L}$, the MFO yields a Cost $_{T}$ of $126.8486 \mathrm{MW}$, which is the most expensive amongst the five methods simulated. In Case 4, PSO still retains its potential as a method that provides the most consistent Cost $_{T}$ results in all 100 simulations conducted, i.e. in the range between 119.6477-121.4457 MW. Overall, WDO tuned 40 generators to produce the lowest values among the best, worst and average Cost $_{T}$ values at 117.8404, 125.0225 and 122.4021 MW, respectively. This makes WDO as the technique of choice in tuning the generator unit in producing the cheapest generation cost according to the $P_{D}$ that has been set with minimum $P_{L}$.

Figure 5 shows the convergence curves of all five optimization techniques which give the best cost $_{T}$ for Case 1. From the figure, EP converge in 16 iterations, followed by PSO (52 iterations), WDO (62 iterations), FPA (69 iterations) and MFO (91 iterations). Although EP gives the fastest technique to converge, it could not give the lowest generation cost compared to the other four methods. On the other hand, WDO, FPA and PSO are converge almost the same number of iterations. From this result, this indicates the WDO period for convergence is within an acceptable range and is almost identical to other techniques such as FPA and PSO.

\section{Conclusion}

This study proposed a power scheduling strategy using WDO to achieve optimal power generation by generator units with minimum power generation cost based on nonconvex problem formulation. The WDO technique was compared with four selected techniques, namely, FPA, MFO, PSO and EP. The 10- and 40-generator systems, each with two different power demands, were selected as test systems and implemented using MATLAB. Results showed that the five approaches tested successfully produced energies that were almost equal to the energy demands, with extremely low power losses. In terms of generation costs, the majority of scenarios demonstrated that WDO outperforms the other four methods in providing lower generation costs for the same energy demand. From the perspective of iteration time, the WDO capability is found to be equivalent to most of the techniques compared, which requires between 50-100 iterations to complete the calculation. In conclusion, WDO is the most accurate technique that can be used in power scheduling for economic dispatch problems in power systems that consider the point-valve effect.

Acknowledgements This study was supported by the Ministry of Education of Malaysia under the Fundamental Research Grant Scheme (FRGS) Project No. FRGS/1/2018/TK04/UKM/02/7.

\section{References}

1. Farag A, Al-Baiyat S, Cheng TC (1995) Economic load dispatch multiobjective optimization procedures using linear programming techniques. IEEE Trans Power Syst 10(2):731-738

2. Jabr RA, Coonick AH, Cory BJ (2000) A homogeneous linear programming algorithm for the security constrained economic dispatch problem. IEEE Trans Power Syst 15(3):930-936

3. Chen CH and Lin CC (2009) Simple particle swarm optimization for economic dispatch with piecewise quadratic fuel cost function. In: IEEE conference on innovative technologies in intelligent systems and industrial applications, Kuala Lumpur, Malaysia

4. Han S, Kim HJ, Lee D (2020) A long-term evaluation on transmission line expansion planning with multistage stochastic programming. Energies 13(8):1899

5. Ramli NF, Kamari NAM, Zulkifley MA, Musirin I (2020) Optimal power scheduling for economic dispatch using moth flame optimizer. Indones J Electr Eng Comput Sci 20(1):379-384

6. Khorramdel B, Zare A, Chung CY, Gavriliadis P (2020) A generic convex model for a chance-constrained look-ahead economic dispatch problem incorporating an efficient wind power distribution modelling. IEEE Trans Power Syst 35(2):873-886

7. Dong R, Wang S (2020) New optimization algorithm inspired by kernel tricks for the economic emission dispatch problem with valve point. IEEE Access 8:16584-16594

8. Mokarram MJ, Gitizadeh M, Niknam T, Niknam S (2019) Robust and effective parallel process to coordinate multi-area economic 
dispatch (MAED) problems in the presence of uncertainty. IET Gener Transm Distrib 13(18):4197-4205

9. Chen J, Imani Marrani H (2020) An efficient new hybrid ICA-PSO approach for solving large scale non-convex multi area economic dispatch problems. J Electr Eng Technol 15:1127-1145

10. Lee S, Shim H (2019) Distributed algorithm for economic dispatch problem with separable losses. IEEE Control Syst Lett 3(3):685-690

11. Yang XS, Karamanoglu M, He X (2013) Multi-objective flower algorithm for optimization. Proced Comput Sci 18:861-868

12. Prasanth Ram J, Rajasekar N (2017) A novel flower pollination based global maximum power point method for solar maximum power point tracking. IEEE Trans Power Electron 32(11):8486-8499

13. Potnuru D, Mary KA, Babu CS (2019) Experimental implementation of flower pollination algorithm for speed controller of a BLDC motor. Ain Shams Eng J 10(2):287-295

14. Peesapati R, Yadav VK, Kumar N (2018) Flower pollination algorithm based multi-objective congestion management considering optimal capacities of distributed generations. Energy 147:980-994

15. Mirjalili S (2015) Moth-flame optimization algorithm: a novel nature-inspired heuristic paradigm. Knowl-Based Syst 89:228-249

16. Hongwei L, Jianyong L, Liang C, Jingbo B, Yangyang S, Kai L (2019) Chaos-enhanced moth-flame optimization algorithm for global optimization. J Syst Eng Electron 30(6):1144-1159

17. Li C, Niu Z, Song Z, Li B, Fan J, Liu PX (2018) A double evolutionary learning moth-flame optimization for real-parameter global optimization problems. IEEE Access 6:76700-76727

18. Kotb MF, El-Fergany AA (2020) Optimal power flow solution using moth swarm optimizer considering generating units prohibited zones and valve ripples. J Electr Eng Technol 15:179-192

19. Kamari NAM, Musirin I, Dagang AN, Zaman MHM (2020) PSObased oscillatory stability assessment by using the torque coefficients for SMIB. Energies 13(5):1-16

20. Hannan MA, Abdolrasol MGM, Faisal M, Ker PJ, Begum RA, Hussain A (2019) Binary particle swarm optimization for scheduling MG integrated virtual power plant toward energy saving. IEEE Access 7:107937-107951

21. Sato M, Fukuyama Y, Iizaka T, Matsui T (2019) Total optimization of energy networks in a smart city by multi-swarm differential evolutionary particle swarm optimization. IEEE Trans Sustain Energy 10(4):2186-2200

22. Tian J, Tan Y, Zeng J, Sun C, Jin Y (2019) Multiobjective infill criterion driven gaussian process-assisted particle swarm optimization of high-dimensional expensive problems. IEEE Trans Evol Comput 23(3):459-472

23. Kamari NAM, Musirin I, Ibrahim AA (2020) Swarm intelligence approach for angle stability improvement of PSS and SVC-based SMIB. J Electr Eng Technol 15:1001-1014

24. Che A, Wu P, Chu F, Zhou MC (2015) Improved quantuminspired evolutionary algorithm for large-size lane reservation. IEEE Trans Syst Man Cybern Syst 45(12):1535-1548

25. Musirin I, Sivaraju SS, Razali NAN, Kamari NAM, Senthil Kumar AV, Othman MM (2021) Hybrid cuckoo search-evolutionary programming technique for distributed generation and battery energy storage installation. Eur J Mol Clin Med 8(2):495-507

26. Gee SB, Tan KC, Abbass HA (2017) A benchmark test suite for dynamic evolutionary multiobjective optimization. IEEE Trans Cybern 47(2):461-472

27. Bayraktar Z, Komurcu M, Werner DH (2010) Wind driven optimization (WDO): a novel nature-inspired optimization algorithm and its application to electromagnetics. IEEE antennas and propagation society international symposium. Ontario, Canada
28. Kusiak A, Zhang Z, Xu G (2013) Minimization of wind farm operational cost based on data-driven models. IEEE Trans Sustain Energy 4(3):756-764

29. Ning C, You F (2019) Data-driven adaptive robust unit commitment under wind power uncertainty: a bayesian nonparametric approach. IEEE Trans Power Syst 34(3):2409-2418

30. Abdella O, Rezk H, Ahmed EM (2019) Wind driven optimization algorithm based global MPPT for PV system under non-uniform solar irradiance. Sol Energy 180:429-444

31. Basu M (2011) Economic environmental dispatch using multi-objective differential evolution. Appl Soft Comput 11(2):2845-2853

Publisher's Note Springer Nature remains neutral with regard to jurisdictional claims in published maps and institutional affiliations.

Nur Fariza Ramli received Bachelor in Electrical and Electronic Engineering from Universiti Teknologi Petronas, Malaysia in 2008. She is currently an executive at Yayasan Pelajaran Mara. Meanwhile, at the same time she is pursuing towards the M.Sc. at Faculty of Engineering and Built Environment, Universiti Kebangsaan Malaysia. Her research interest are energy management and artificial intelligence.

Nor Azwan Mohamed Kamari received Bachelor in Electrical and Electronic Engineering from Meiji Univeristy, Japan, M.Sc. in Electrical and Electronic Engineering from Ehime University, Japan and Ph.D. in Electrical Engineering from Universiti Teknologi Mara, Malaysia in 2000, 2004 and 2016, respectively. He has been an academician for over 10 years at several universities before joining Faculty of Engineering and Built Environment, Universiti Kebangsaan Malaysia (UKM) in 2017. His areas of research interests are power engineering, energy management and artificial intelligence.

Syahirah Abd Halim received the bachelor's degree in Electrical \& Electronics Engineering from Universiti Teknologi Petronas, Malaysia in 2009. She received the master's degree and the $\mathrm{PhD}$ degree in Electrical Engineering from the University of Malaya, Malaysia in 2012 and 2016, respectively. She joined UM Power Energy Dedicated Advanced Centre as a Postdoctoral Research Fellow in January 2017. Since August 2017, she has been a Senior Lecturer with the Faculty of Engineering and Built Environment, Universiti Kebangsaan Malaysia. Her research interests include transmission line modelling, transient analysis and electrical grounding techniques.

Mohd Asyraf Zulkifley received the B.Eng. degree in mechatronics from International Islamic University Malaysia in 2008 and the Ph.D. degree in electrical and electronic engineering from The University of Melbourne, Australia in 2012. He was a sponsored Researcher at the Department of Computer Science, University of Oxford from 2016 to 2018. He is currently an Associate Professor at the Faculty of Engineering and Built Environment, Universiti Kebangsaan Malaysia. His current research interests are visual object tracking and medical image analysis.

Mohd Saiful Mohd Sahri received Bachelor in Electronics and Computer System Engineering and Master in Electronics and Computer System Engineering from Takushoku Univeristy, Japan in 2007 and 2009 , respectively. He is currently a senior lecturer at Universiti Kuala Lumpur Malaysia France Institute. His current research interests are superconductor application and artificial intelligence.

Ismail Musirin obtained Bachelor of Electrical Engineering (Hons) in 1990 from Universiti Teknologi Malaysia, M.Sc. Pulsed Power Technology in 1992 from University of Strathclyde, United Kingdom and 
Ph.D. in Electrical Engineering from Universiti Teknologi MARA (UiTM), Malaysia in 2005. He is currently a Professor of Power System at the Faculty of Electrical Engineering, UiTM and headed the Power System Operation (POSC) Computational Intelligence Research Group. He has authored 2 books published, over 300 papers in international indexed journals and conferences. He is also an international journal reviewer for IEEE Transactions, Elsevier Science, WSEAS, John Wiley, IET and some other publishers. He has chaired more than 20 international conferences since 2007. To date, he has delivered keynote speeches at Cambridge University, United Kingdom, Dubai, Korea and Malaysia. He has also been given opportunity to evaluate research grants at the national and international levels. His research interest includes artificial intelligence, optimization techniques, power system analysis, renewable energy, distributed generation and power system stability. He is a professional engineer and a senior member of International Association of Computer Science and Information Technology (IACSIT), member of Artificial Immune System Society (ARTIST) and member of International Association of Engineers (IAENG), Hong Kong. 